\title{
Sex Hormones Fluctuation and Obsessive Compulsive Disorder
}

\section{Journal of \\ Neurology and Psychology}

\author{
João Alcafache* \\ Department of Psychiatry and Mental Health, Baixo Vouga Hospital \\ Centre, Portugal \\ *Address for Correspondence \\ João Alcafache, MD, Department of Psychiatry and Mental Health, Baixo \\ Vouga Hospital Centre, Av. Artur Ravara, 3814-501 Aveiro, Portugal, E-mail: \\ jalcafachef@sapo.pt \\ Submission: 02 November, 2015 \\ Accepted: 30 January, 2016 \\ Published: 05 February, 2016 \\ Copyright: (c) 2016 Alcafache J. This is an open access article \\ distributed under the Creative Commons Attribution License, which \\ permits unrestricted use, distribution, and reproduction in any medium, \\ provided the original work is properly cited. \\ Reviewed \& Approved by: Dr. Tammy J Jechura, Psychological \\ Science, Albion College, USA
}

Anecdotal findings suggests that it's not unusual in the daily practice of physicians to hear complaints of a mother's fear of hurting her new born child by an accidentally cutting the child with a knife, throwing him out of a window, or contaminating him with a disease or a toxic product. Thus, this is not just a matter of the psychiatry specialty, but concerns all the specialties in medicine. In the majority of cases, even without being aware of a diagnosis, there is an underlying OCD spectrum condition [4]. The early detection of these conditions and quicker reference to specialty treatment can change the patient's quality of life and even change the course of the disease.

OCD is a severe psychiatric condition with a global estimated prevalence of $2 \%-3 \%$ [5], affecting all age and culture groups [6]. The onset age of OCD in male population is significantly earlier than in female one [7], although it remains unclear if there is a higher prevalence in one specific gender, as the prevalence female/male ratio ranges from 0.8 to 3.8 [8]. In women, the onset age is bimodal, with the first peak between 13 and 16 years and the second peak almost a decade later, between 22 and 32 years. These peaks coincide with puberty and child-bearing years, respectively [9]. Besides occurring during these two main life periods, other phases related to the reproductive cycle have also been associated with an increased risk of onset or exacerbation of OCD: premenstrual, pregnancy and postpartum $[4,10,11]$.

Many studies have been recently conducted in the area of sex hormones and OCD, but they have not significantly impacted the way OCD is treated. In this paper, the authors review the available information addressing the relationship between reproductive hormones and OCD. We specifically address the following topics: i) the relationship between OCD onset or worsening and the fluctuation of hormone levels; ii) women's increasing propensity to develop OCD; and iii) future diagnosis and treatment approaches.

\section{Gender-related hormones and mental disorders}

Sex hormones are chemical substances produced in the gonads 
(endocrine glands) and directly secreted into the bloodstream. The most relevant sex hormones are testosterone (it is part of a group of hormones: the androgens), estrogens, and progesterone. The regulation of these hormones is dependent on the respective gonads (testicles and ovaries) that are, in turn, also dependent on other glands: the pituitary gland and a closely related region of the brain, the hypothalamus, which regulates the pituitary itself. These three structures, the hypothalamus, pituitary, and gonads have a determinant influence in global sexual functions such as the menstrual cycle, pregnancy, changes of puberty and sexual behavior [12].

Assuming a regular 28-day cycle, the levels of sex hormones in the female body fluctuate according to five general phases. In phase 1, the menstrual phase (day 1 to 5 of the cycle), when secretion of estrogen occurs, the secretion of progesterone ceases. During phase 2, the follicular phase (day 6 to 12), estrogen levels increase progressively and there is almost no progesterone. In phase 3, the peri-ovulatory phase (day 13 to 15), estrogen levels decrease and progesterone levels are also very low. In phase 4 , the luteal phase (day 16 to 23), progesterone is secreted in huge quantities, and the estrogen levels achieve a second peak. Finally, in phase 5 , the premenstrual phase (day 24 to 28), both estrogen and progesterone levels decline dramatically (Figure 1). Levels of testosterone are virtually constant [13].

The effects of sex hormones are not confined to the reproductive system. The pharmacological effects of neurosteroids in the brain have been extensively studied concerning their anti-convulsant [14], anti-depressant [15], and anti-anxiety properties [16]. These "psychopharmacological" properties were attributed by Rupprecht et al. to the modulation of neuronal plasticity via genomic actions [17]. Compagnone and Mellon attributed these effects to the bidirectionalallosteric modulation of $\gamma$-aminobutyric-A (GABA A) receptors and NMDA-preferring glutamate receptors [18] confirming that allopregnanolone and DHEA/DHEAS have opposite effects on GABA A and NMDA receptors [19].

These findings could be important when considering treatment of OCD. The usual treatment of OCD consists of high doses of SSRIs (much higher than antidepressant doses), and when the

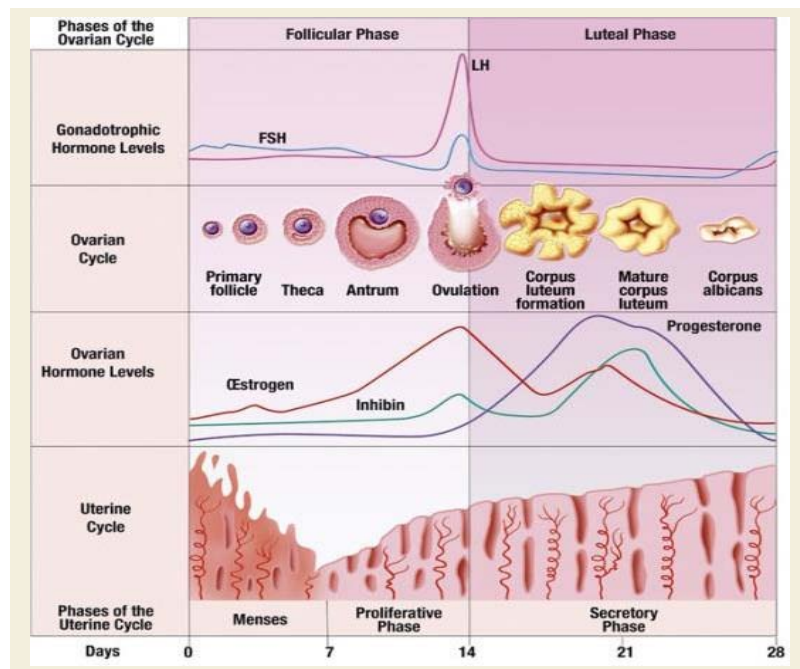

Figure 1: Regular female hormonal 28-day cycle. basic treatment fails, before thinking of invasive solutions (such as Deep Brain Stimulation to interrupt circuit loops involved in the pathophysiology of OCD), argumentation strategies are often used in the non-responders by adding D2 Blockers in low doses, such as haloperidol, risperidone, olanzapine, among others.

These strategies are supported by other relevant findings which were reported by Jaworska-Feil et al. about the action on GABA A receptors of neurosteroids in central dopaminergic transmission at the nucleus accumbens [20]. Robichaud and Debonnel also reported that allopregnanolone increases firing rate of raphe nuclei serotonergic neurons [21], suggesting a possible explanation to the augmentation strategy with D2 blockers in OCD treatment-resistant patients.

Progesterone and other steroid hormones act through genomic (classical) and non-genomic (non-traditional) mechanisms, which induce delayed and immediate effects, respectively $[22,23]$. The two independent pathways of action can explain some of the delayed obsessive-compulsive symptoms when considering hormonal fluctuations. This differential effect was also reported to interfere with OCD symptoms, as Flaisher-Grinberg et al. reported. This study refers that acute administration of estradiol 24 hours before an obsessive-compulsive simulation test in pre-pubertal female rats significantly decreased the number of compulsive behaviors, while no anti-compulsive effect was observed when estradiol was administered only 1 hour before the test [24]. These findings suggest that the anti-compulsive effect of estradiol could be mediated by genomic changes induced by estradiol. In fact, as already described by Stein, Friedlander and Benmansour, neurosteroids, particularly estradiol, can interact with several neural systems implicated in the pathophysiology of OCD, including the serotonergic system, the dopaminergic system and the orbitofrontal cortex [25-30], which are the two most important systems involved in the actual treatment of the disease (SSRIs and D2 blockers), and major brain areas also involved in past and present invasive therapies (DBS and psychosurgery).

\section{Gender-related hormones and OCD}

OCD symptoms follow a fluctuating pattern, as sex hormones do, so it becomes interesting to understand how these fluctuations can overlap each other. Thus, the first research approaches on these matters consisted of studying the effects of sex hormones in the brain, specifically in certain areas known as having impaired functioning in OCD patients [26,31]. It is well documented that estrogen has multiple neuromodulating actions, either by direct intracellular action $[32,33]$ or by mediation of numerous neurotransmitter systems in the central nervous system (CNS). Actions on serotonergic, adrenergic, and cholinergic neurons, pathways, and receptors are well-studied $[32,34]$. Less is known about progesterone.

It's not rare for a general practitioner, a neurologist, a cardiologist, or any other physician, to discover "tick-like" behaviors, such as nail biting, pulling hair, etc., in anxious patients who request an appointment for other somatic complaints. Similar behavior was studied in animal models, which provided further insight into the role of gonadal hormones (or their precursors) in OCD and OCD spectrum conditions. 


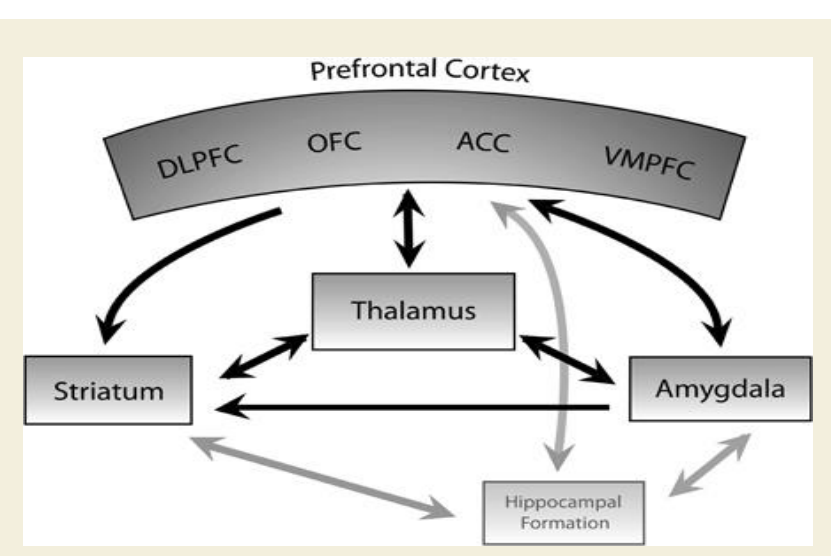

Figure 2: Cortico-striato-thalamocortical circuit. Schematic diagram of the cortico-striato-thalamocortical (CSTC) circuit, which is implicated in the pathophysiology of OCD. In DBS for OCD, activity within this circuit is thought to be modulated by high frequency stimulation.

DLPFC: Dorsolateral Prefrontal Cortex; ACC: Anterior Cingulate Cortex; OFC: Orbitofrontal Cortex; VMPFC: Ventromedial Prefrontal Cortex. Arrowheads indicate direction of neural input. Black lines represent connections among the major structures implicated in DBS for OCD; gray lines indicate additional connections. (Adapted from Mechanisms of deep brain stimulation for obsessive compulsive disorder: effects upon cells and circuits. Sarah K. Bourne et al. 2012)

Many of them employed the 'marble-burying behavior' of mice as a predictor of anti-OCD effect, since it is considered to have a significant predictive validity [35]. Umathe et al. studied the effect of allopregnanolone (a neurosteroid, precursor of progesterone), dehydroisoandrosterone 3-sulphate (DHAS), progesterone and finasteride in marble-buryingbehavior of mice, using a classic control with fluoxetine (a typical SSRI used in the treatment of OCD) [19]. The authors concluded that allopregnanolone attenuated marbleburying behavior and spontaneous alternation behavior, whereas DHAS had opposite effects, exacerbating the symptoms, suggesting that neurosteroids could be an effective target in the management of OCD [19]. These findings open the door to another possible pharmacologic group in the treatment approach of an OCD patient, such as an augmentation strategy.

Anti-androgen effects on OCD were also studied, even though in a small scale. One of the studies was conducted with flutamide, a synthetic, non-steroidal, competitive antagonist of the androgen receptor. They concluded that there was a lack of response to treatment with flutamide, which, in the opinion of the authors, suggests that any effects of gonadal steroids to exacerbate OCD symptoms are more likely to be mediated by estrogen receptors, rather than by mechanisms involving classical intracellular androgen receptors [36]. However, results from studies on the anti-androgen effects are contradictory. One study using a long-acting analogue of gonadotropin-releasing hormone, which is known to reduce levels of circulating androgens to almost zero, showed a great improvement in OCD symptoms, after conventional pharmacology was tested [37]. Research has also shown a positive result using cyproterone acetate in ordinary doses for several months [38].

Pinna et al. reported that a well-known "anti-obsessive" compound, fluoxetine, increased the levels of allopregnanolonein the brain at a dose that is ten-fold lower than that required to block serotonin reuptake, which reinforces Umathe's results in animal studies [19]. Bigos et al. in 2009, confirmed the deregulation of neurosteroids in OCD patients, although the study presentssome limitations, such as a small sample size [39]. These studies suggest that could be linkage between the neurosteroid and the serotoninergic systems, probably misregulated in OCD patients.

Hill et al. reported that estrogen-deficient mice (aromatase knockout) showed increased wheel running, self-grooming, and barbering (similar to obsessive-compulsive symptoms), when compared to normal type mice [40]. Thus, we are able to presume that, in phases of the menstrual cycle or in the reproductive events, when the estrogen levels significantly decrease, women are at risk of developing OCD symptoms. This begs the question of whether we should prevent symptoms with SSRI treatment, birth control, or hormonal intervention.

\section{Women: more hormones, more risk?}

The studies to date suggest that women are an at-risk population, or at least, they pass through phases that represent more risk. In a study with 90 women with OCD, it was reported that OCD onset within the year of menarche was $21.1 \%$ [41]. On the other hand, onset of OCD related to the birth of a child (not necessarily the first) has been classically considered to be around 3-17\% for all patients (man and women) and at $13-27 \%$ when considered only female patients [10].

The factors that could be related to OCD after delivery were also explored. When obstetric complications were assessed, post-partum OCD was found to be more frequent in primiparae $(75 \%)$ cases, frequently follows a pre- and post-term pregnancy $(62.5 \%)$, and is related to caesarean without labor $(62.5 \%)$ [11].

OCD onset has been reported to be significantly lower in menopause (2\%) than in menarche or childbirth [41]. Williams et al. observed that OCD symptoms worsened in $17 \%$ of patients during pregnancy, $25 \%$ at post-partum, and $42 \%$ at premenstruum [42]. However these data are widely variable across the literature, with reported worsening rates during pregnancy varying from $8 \%$ to $46 \%[4,10,41,43,44]$. Uguz et al. reported a worsening rate of 32 , $7 \%$, relating that to comorbid major depression and anxiety disorder during pregnancy [45].

When comparing these data with some recent neuroimagiologic findings, we once again find a relationship between hormone fluctuation and OCD findings. In an fMRI study, it was proposed that OCD symptom dimensions are mediated by different components of frontostriatal-thalamic circuits involved in cognitive and emotional processing [46], specifically the areas related to the contamination/ washing dimensions: bilateral ventromedial prefrontal regions and right caudate nucleus [46]. These areas have larger volumes in women than in men and also have high levels of sex steroid hormone receptors, showing sexual dimorphism [47].

Considering all the available accumulated data outlined here, it appears as though perhaps women are at more risk for OCD symptoms than men, not only by hormonal profile, but also due to structural brain dimorphisms in areas linked to fear, responsibility, 
and social behavior. The data suggest that an integration of biological and cognitive theories for OCD an etiology.

\section{Only a matter of OCD or a matter of all psychiatry?}

Many studies have suggested that the relevant biographic moments of a woman's life, the reproductive ones, have an increased risk of producing psychiatric conditions $[9,48]$. However this is not exclusive of the female gender; these are also risky life moments for male individuals who are not exposed to the hormonal fluctuations. There are very few studies about this specific topic; however we found a limited but interesting article about acute onset of obsessivecompulsive disorder in males. In this work, the authors described four case reports of patients, assessed at different clinics in different geographical regions, who presented obsessive-compulsive thoughts about their fear of harming the new-born child or pregnant wife. This report suggests that pregnancy and the post-partum period are also vulnerable periods for the development of OCD in expecting fathers [49].

One could attribute these findings to stress and likely to cortisol. However, it is difficult to differentiate whether only negative events have these effects or if positive events also cause them. It can be difficult to catalogue an event such as menarche or menopause as negative or positive. Some authors have proposed that even positive life events act as additional stressors rather than buffers, proposing a chaotic life course model [50]. According to this hypothesis, Overbeek et al. when studying the impact of life's stressful events in affective disorders, proposed that positive events were not found to have a buffer impact on negative life events. On the other hand, when positive life events combine with negative life events to create an erratic period, they could represent an even greater threat to an individual's mental health [50].

More information is available for the effects of life events on depression and psychosis, but there is less or none for OCD. Testing the theory that these life event effects are related to anxiety, and anxiety to cortisol release profile, Het et al. studied stress-induced cortisol elevations, and suggested that cortisol is associated with an attenuated negative emotional arousal in response to acute stress. This seems to support the hypothesis of mood-buffering effects of cortisol [51].

Pruessner et al. studied the effect of cortisol in individuals at ultra-high risk for psychosis (reported by the same authors to have elevated levels of chronic stress and deficits in coping skills). They found weakened cortisol responses to acute psychosocial stress, which was attributed to high chronic stress and desensitization of the hypothalamic-pituitary-adrenal axis [52].

All these studies have shown that there is no clarified consensus around the theme of stress, cortisol, and increased risk for psychiatric impairment, so it's even harder to predict if reproductive stressful biographic events worsen obsessive-compulsive symptoms or are even enablers of OCD onset. Some authors state that circadian cortisol levels are higher in OCD patients, however contradictory results were found in stress response tests $[53,54]$.

To address this issue, a study was designed in which OCD patients were submitted to exposure with response prevention (ERP is an established treatment for patients with OCD. Exposure means confronting one's fears repeatedly until the fear subsides, while response prevention means refraining from compulsions, avoidance, or escape behaviors)and after that, measures of salivary cortisol and subjective units of distress (SUD) were taken. It was concluded that despite considerable psychological stress, as measured by the constant increase in SUD, no increase in cortisol was observed [55].

Since cortisol does not seem to be the answer to a possible hormonal impairment in the aethiology OCD, the response of cortisol was studied in parallel with another infrequently explored hormone, salivary $\alpha$-amylase (sAA), which serves as a marker of sympatho-adrenal medullary system (SAM) activity in OCD. After submitting OCD patients and controls to an electrical stimulation stress, they measured the responsiveness of these two hormones and found that SAA levels in OCD patients were significantly elevated in relation to controls before and after electrical stimulation, but found no differences in salivary cortisol changes, when comparing OCD patients and controls [56].

A pilot study was conducted to evaluate the effects of stress in cortisol and AA on a major reproductive period, namely the postpartum period. Stress response was studied in post-partum women with OCD, submitting them to a physiological stress test, the Cold Pressor Test (CPT), in comparison to healthy controls, and cortisol and AA were measured. OCD women did not show higher cortisol or AA responses to stress when compared to healthy controls, whereas OCD women showed larger variability in the AA and cortisol responses following the CPT [57].

\section{Alternative explanations: Non-steroid hormones: Oxytocin?}

Studies published in the last few years have tried to investigate oxytocin's relevance on several human behaviors, such us orgasm, social recognition, pair bonding, anxiety, and parenthood [58]. In this particular case, motherhood, oxytocin showed to be critically involved in the initiation of maternal behavior in animals [59], with greater elevation of its levels in the cerebrospinal fluid during the last trimester of pregnancy and the post-partum period. In addition, animal models also show an increase in oxytocin messenger ribonucleic acid in the female brain during puberty [60].

It was proposed that a sudden high increase in oxytocin near the end of pregnancy and during the puerperium may act as a trigger for an exacerbation or the onset of OCD. The authors argued that oxytocin could play a role in the pathogenesis of obsessive-compulsive sexual thoughts/images which are quite common obsessions in nonpregnant OCD patients [61-63]. However, further investigation is needed on the relationship between OCD and oxytocin.

\section{Conclusion}

Due to the high prevalence of OCD, OCD patients are treated by many different medical specialties besides psychiatry and many symptoms are misdiagnosed and attributed to stress or other conditions. In this article, we argue that it is clear that there are specific life periods with increased risk of onset of OCD symptoms/ condition; periods that deserve special attention from all clinicians to aid in the diagnosis and treatment of OCD. 
There is also an unequivocal relationship between relevant gonadal fluctuation periods and the worsening or onset of OCD, which generally coincides with major stressful periods. This could help explain a mechanism of action believed to be related to cortisol metabolism in other significant psychiatric conditions (e.g. depression, psychosis, etc.). However, the impact of these stressful periods in OCD is even greater, with much higher percentages, without producing a substantial variation in cortisol blood levels.

These findings open the door to an alternate explanation, possibly linked to the direct or indirect effect of gonadal hormones and some of their metabolites (neurosteroids) on specific brain receptors located in specific areas known for being implicated in OCD.

The particular events in women's gonadal biography that present an increased risk for OCD onset or worsening are menarche, pregnancy, the puerperium period, and, for some authors, menopause. In our review, we found values for OCD onset or exacerbation of symptoms of $21 \%$ for menarche, $13-27 \%$ for pregnancy, $29 \%$ for puerperium, and $2 \%$ for menopause. Empirically, we can see that the closer to the evolutionary act of passing the genes to the next generation, the higher are the risks for OCD, with a percentage similar to the general population at menopause. When plotted on a graph, these particular events compose an inverse U curve in women's reproductive cycle of life. We should also highlight the relatively high percentages of worsening of OC symptoms in the premenstruum period, which can be as high as $42 \%$. However, in the studies that assess these percentages at premenstruum, the period of 14 days before catamenia is too large, which makes the values more likely to be affected by premenstrual dysphoric disorder. Dividing this phase would allow us to better study the curve within each menstrual cycle and determine whether or not it is also an " $n$ " curve. This could lead to an eventual co-therapy with specific oral contraceptive drugs for resistant cases.

The gold standard treatment for OCD consists of the use of high doses of Selective Serotonin Reuptake Inhibitors (SSRIs) and Tricyclic Antidepressants (mainly clomipramine), with or without psychotherapy support (manly CBT), with response rates ranging from $60 \%$ to $80 \%$ [64]. This means that $20-40 \%$ of OCD patients are partial responders or non-responders [65-67]. Many circumstances and/or characteristics of the disorder may be related to this unpredictable response, such as symptom severity, age at onset, course of the disease, family functioning, metabolic capacity, neurobiology, brain development and genetic background $[68,69]$. Taking the serotonin hypothesis of OCD as the most promising one in the pathophysiology of this disease [70], many authors proposed that an acute onset of symptoms during pregnancy or the puerperium period are due to quick changes in levels of reproductive hormones (i.e., estrogen and progesterone), which in turn influence serotonin levels [71]. At this time, the exact neurobiological mechanisms implicit in the onset of OCD have not been clearly demonstrated and research does not assume that OCD is due to a dysfunctional serotonin system [72]. Many others suggest that the symptoms of OCD could be related to interactions between gonadal hormones rapidly fluctuating and the metabolism of GABA and dopamine.

All the studies examined in this review show that the gonadal hormones, estrogen and progesterone, , represent an increased risk for OCD onset or worsening during the puerperium period, with some authors assuming that gonadal hormones may change serotoninergic function [73]. In spite of not knowing the exact mechanism of action, we can observe that the periods of gonadal hormone fluctuations coincide with worsening and onset of OCD. The high incidence of the disease in significant life events for women (28-64\%) constitutes a serious issue [74]. Therefore, an aggregative theory is needed to explain and integrate all these findings.

\section{References}

1. World Health Organization (2007) Gender and women's mental health. Gender disparities and mental health: The facts.

2. Santvana S, Shamsah. S, Firuza P, Rajesh P (2005) Psychiatric disorders associated with pregnancy. J Obstet Gynecol India 55: 218-227.

3. Chung WC, Auger AP (2013) Gender differences in neurodevelopment and epigenetics. Pflugers Arch 465: 573-584.

4. Glazier K, Swing M, McGinn LK (2015) Half of obsessive-compulsive disorder cases misdiagnosed: vignette-based survey of primary care physicians. J Clin Psychiatry 76: e761-e777.

5. Rasmussen SA, Eisen JL (1992) The epidemiology and differential diagnosis of obsessive compulsive disorder. J Clin Psychiatry 53 Suppl: 4-10.

6. Bartz JA, Hollander E (2006) Is obsessive-compulsive disorder an anxiety disorder? Prog Neuropsychopharmacol Biol Psychiatry 30: 338-352.

7. Bogetto F, Venturello S, Albert U, Maina G, Ravizza L (1999) Gender-related clinical differences in obsessive-compulsive disorder. Eur Psychiatry 14: 434441.

8. Weissman MM, Bland RC, CaninoGJ, Greenwald S, Hwu HG, et al. (1994) The cross national epidemiology of obsessive compulsive disorder. The Cross National Collaborative Group. J Clin Psychiatry 55 Suppl: 5-10.

9. Brandes M, Soares CN, Cohen LS (2004) Postpartum onset obsessivecompulsive disorder: diagnosis and management. Arch Womens Ment Health 7: $99-110$.

10. Labad J, Menchon JM, Alonso P, Segalas C, Jimenez S, et al. (2005) Female reproductive cycle and obsessive-compulsive disorder. J Clin Psychiatry 66: 428-435.

11. Maina G, Albert U, Bogetto F, Vaschetto P, Ravizza L (1999) Recent life events and obsessive-compulsive disorder OCD : the role of pregnancy/ delivery. Psychiatry Res 89: 49-58.

12. Hyde J, DeLamater J (2013) Understanding human sexuality, $12^{\text {th }}$ Edition.

13. Buser T (2009) The impact of female sex hormones on competitiveness.

14. Kokate TG, Svensson BE, Rogawski MA (1994) Anticonvulsant activity of neurosteroids: correlation with gamma-aminobutyric acid-evoked chloride current potentiation. J Pharmacol Exp Ther 270: 1223-1229.

15. Khisti RT, Chopde CT, Jain SP (2000) Antidepressant-like effect of the neurosteroid 3alpha-hydroxy-5alpha-pregnan-20-one in mice forced swim test. Pharmacol Biochem Behav 67: 137-143.

16. Barbaccia ML (2004) Neurosteroidogenesis: relevance to neurosteroid actions in brain and modulation by psychotropic drugs. Crit Rev Neurobiol 16: $67-74$.

17. Rupprecht R, Reul JM, Trapp T, van Steensel B, Wetzel C, et al. (1993) Progesterone receptor-mediated effects of neuroactive steroids. Neuron 11: 523-530.

18. Compagnone NA, Mellon SH (2000) Neurosteroids: biosynthesis and function of these novel neuromodulators. Front Neuroendocrinol 21: 1-56.

19. Umathe SN, Vaghasiya JM, Jain NS, Dixit P (2009) Neurosteroids modulate compulsive and persistent behavior in rodents: Implications for obsessivecompulsive disorder. Prog Neuropsychopharmacol Biol Psychiatry 33: 11611166.

20. Jaworska-Feil L, Budziszewska B, Leśkiewicz M, Lasoń W, et al. (1998) 
Opposite effects of inhibitory and excitatory neurosteroids on [3H]dopamine release from rat nucleus accumbens. Pol J Pharmacol 50: 449-452.

21. Robichaud M, Debonnel G (2006) Allopregnanolone and ganaxolone increase the firing activity of dorsal raphe nucleus serotonergic neurons in female rats. Int J Neuropsychopharmacol 9: 191-200.

22. Brann DW, Mahesh VB (1995) Glutamate: a major neuroendocrine excitatory signal mediating steroid effects on gonadotropin secretion. J Steroid Biochem Mol Biol 53: 325-329.

23. McEwen BS, Alves SE (1999) Estrogen actions in the central nervous system. Endocr Rev 20: 279-307.

24. Flaisher-Grinberg S, Albelda N, Gitter L, Weltman K, Arad M, et al. (2009) Ovarian hormones modulate 'compulsive' lever-pressing in female rats. Horm Behav 55: 356-365.

25. Stein DJ (2000) Neurobiology of the obsessive-compulsive spectrum disorders. Biol Psychiatry 47: 296-304.

26. Friedlander L, Desrocher M (2006) Neuroimaging studies of obsessivecompulsive disorder in adults and children. Clin Psychol Rev 26: 32-49.

27. Benmansour S, Piotrowski JP, Altamirano AV, Frazer A (2009) Impact of ovarian hormones on the modulation of the serotonin transporter by fluvoxamine. Neuropsychopharmacology 34: 555-564.

28. Karakaya S, Kipp M, Beyer C (2007) Oestrogen regulates the expression and function of dopamine transporters in astrocytes of the nigrostriatal system. $J$ Neuroendocrinol 19: 682-690.

29. Quinlan MG, Hussain D, Brake WG (2008) Use of cognitive strategies in rats: the role of estradiol and its interaction with dopamine. Horm Behav 53: 185

30. Dreher JC, Schmidt PJ, Kohn P, Furman D, Rubinow D, et al. (2007) Menstrual cycle phase modulates reward-related neural function in women. Proc Natl Acad Sci U S A 104: 2465-2470.

31. Szeszko PR, Ardekani BA, Ashtari M, Malhotra AK, Robinson DG, et al. (2005) White matter abnormalities in obsessive-compulsive disorder: a diffusion tensor imaging study. Arch Gen Psychiatry 62: 782-790.

32. McEwen B (2002) Estrogen actions throughout the brain. Recent Prog Horm Res 57: 357-384

33. Joffe H, Cohen LS (1998) Estrogen, serotonin, and mood disturbance: where is the therapeutic bridge? Biol Psychiatry 44: 798-811.

34. Stahl SM (1998) Basic psychopharmacology of antidepressants, part 2: Estrogen as an adjunct to antidepressant treatment. J Clin Psychiatry 59 Suppl 4: 15-24

35. Joel D (2006) Current animal models of obsessive compulsive disorder: a critical review. Prog Neuropsychopharmacol Biol Psychiatry 30: 374-388.

36. Altemus M, Greenberg BD, Keuler D, Jacobson KR, Murphy DL (1999) Open trial of flutamide for treatment of obsessive-compulsive disorder. J Clin Psychiatry 60: 442-445

37. Eriksson $T$ (2000) Antiandrogenic treatment for obsessive-compulsive disorder. Am J Psychiatry 157: 483

38. Casas M, Alvarez E, Duro P, Garcia-Ribera C, Udina C, et al. (1986) Antiandrogenic treatment of obsessive-compulsive neurosis. Acta Psychiat Scand 73: 221-222.

39. Bigos KL, Folan MM, Jones MR, Haas GL, Kroboth FJ, et al. (2009) Dysregulation of neurosteroids in obsessive compulsive disorder. J Psychiat Res 43: 442-445.

40. Hill RA, Mclnnes KJ, Gong EC, Jones ME, Simpson ER, et al. (2007) Estrogen deficient male mice develop compulsive behavior. Biol Psychiatry 61: 359-366.

41. Labad J, Alonso P, Segalas C, Real E, Jimenez S, et al. (2010) Distinct correlates of hoarding and cleaning symptom dimensions in relation to onse of obsessive-compulsive disorder at menarche or the perinatal period. Arch Womens Ment Health 13: 75-81.
42. Williams KE, Koran LM (1997) Obsessive-compulsive disorder in pregnancy, the puerperium, and the premenstruum. J Clin Psychiatry 58: 330-334.

43. Uguz F, Gezginc K, Zeytinci IE, Karatayli S, Askin R, et al. (2007) Obsessivecompulsive disorder in pregnant women during the third trimester of pregnancy. Compr Psychiatry 48: 441-445.

44. Forray A, Focseneanu M, Pittman B, McDougle CJ, Epperson CN (2010) Onset and exacerbation of obsessive-compulsive disorder in pregnancy and the postpartum period. J Clin Psychiatry 71: 1061-1068.

45. Uguz F, Kaya V, Gezginc K, Kayhan F, Cicek E (2011) Clinical correlates of worsening in obsessive-compulsive symptoms during pregnancy. Gen Hosp Psychiatry 33: 197-199.

46. Mataix-Cols D, Wooderson S, Lawrence N, Brammer MJ, Speckens A, et al. (2004) Distinct neural correlates of washing, checking, and hoarding symptom dimensions in obsessive-compulsive disorder. Arch Gen Psychiatry 61: 564-576.

47. Goldstein JM, Seidman LJ, Horton NJ, Makris N, Kennedy DN, et al. (2001) Normal sexual dimorphism of the adult human brain assessed by in vivo magnetic resonance imaging. Cereb Cortex 11: 490-497.

48. Ross LE, McLean LM (2006) Anxiety disorders during pregnancy and the postpartum period: A systematic review. J Clin Psychiatry 67: 1285-1298.

49. Abramowitz J, Moore K, Carmin C, Wiegartz PS, Purdon C (2001) Acute onset of obsessive-compulsive disorder in males following childbirth. Psychosomatics 42: 429-431.

50. Overbeek G, Vermulst A, de Graaf R, Ten Have M, Engels R, et al. (2011) Positive life events and mood disorders: longitudinal evidence for a chaotic life-course hypothesis. Tijdschr Psychiatr 53: 321-332.

51. Het S, Schoofs D, Rohleder N, Wolf OT (2012) Stress-induced cortiso level elevations are associated with reduced negative affect after stress: indications for a mood-buffering cortisol effect. Psychosom Med 74: 23-32.

52. Pruessner M, Béchard-Evans L, Boekestyn L, lyer SN, Pruessner JC, et al. (2013) Attenuated cortisol response to acute psychosocial stress in individuals at ultra-high risk for psychosis. Schizophr Res 146: 79-86.

53. Kluge M, Schüssler $P$, Künzel HE, Dresler M, Yassouridis A, et al. (2007) Increased nocturnal secretion of $\mathrm{ACTH}$ and cortisol in obsessive compulsive disorder. J Psychiatr Res 41: 928-933.

54. Gustafsson PE, Gustafsson PA, Ivarsson T, Nelson N (2008) Diurnal cortiso levels and cortisol response in youths with obsessive-compulsive disorder. Neuropsychobiology 57: 14-21.

55. Kellner M, Wiedemann K, Yassouridis A, Muhtz C (2012) Non-response of cortisol during stressful exposure therapy in patients with obsessivecompulsive disorder--preliminary results. Psychiatry Res 199: 111-114.

56. Kawano A, Tanaka Y, Ishitobi Y, Maruyama Y, Ando T, et al. (2012) Salivary alpha-amylase and cortisol responsiveness following electrical stimulation stress in obsessive-compulsive disorder patients. Psychiatry Res 209: 85-90.

57. Lord C, Hall G, Soares CN, Steiner M (2011) Physiological stress response in postpartum women with obsessive-compulsive disorder: A pilot study. Psychoneuroendocrinology 36: 133-138.

58. Lee HJ, Macbeth AH, Pagani JH, Young WS 3rd (2009) Oxytocin: the great facilitator of life. Prog Neurobiol 88: 127-151.

59. Pedersen CA, Prange AJ Jr (1979) Induction of maternal behavior in virgin rats after intracerebroventricular administration of oxytocin. Proc Natl Acad Sci U S A 76: 6661-6665.

60. Miller FD1, Ozimek G, Milner RJ, Bloom FE (1989) Regulation of neuronal oxytocin mRNA by ovarian steroids in the mature and developing hypothalamus. Proc Natl Acad Sci U S A 86: 2468-2472.

61. McDougle CJ, Barr LC, Goodman WK, Price LH (1999) Possible role of neuropeptides in obsessive compulsive disorder. Psychoneuroendocrinology 24: 1-24.

62. Leckman JF, Goodman WK, North WG, Chappell PB, Price LH, et al. (1994) The role of central oxytocin in obsessive compulsive disorder and related normal behavior. Psychoneuroendocrinology 19: 723-749. 
63. Salzberg AD, Swedo SE (1992) Oxytocin and vasopressin in obsessivecompulsive disorder. Am J Psychiatry 149: 713-714.

64. Koran LM, Hanna GL, Hollander E, Nestadt G, Simpson HB, et al. (2007) Practice guideline for the treatment of patients with obsessive-compulsive disorder. Am J Psychiatry 164(7 Suppl): 5-53.

65. Ferrão YA, Shavitt RG, Bedin NR, de Mathis ME, Carlos Lopes A, et al. (2006) Clinical features associated to refractory obsessive-compulsive disorder. J Affect Disord 94: 199-209.

66. DeVeaugh-Geiss J, Katz R, Landau P, Goodman W, Rasmussen S (1990) Clinical predictors of treatment response in obsessive compulsive disorder: exploratory analyses from multicenter trials of clomipramine. Psychopharmacol Bull 26: 54-59.

67. de Haan E, van Oppen $P$, van Balkom AJ, Spinhoven P, Hoogduin KA, et al. (1997) Prediction of outcome and early vs. late improvement in OCD patients treated with cognitive behaviour therapy and pharmacotherapy. Acta Psychiatr Scand 96: 354-361.

68. Leckman JF, Denys D, Simpson HB, Mataix-Cols D, Hollander E, et al (2010) Obsessive-compulsive disorder: a review of the diagnostic criteria and possible subtypes and dimensional specifiers for DSM-V. Depress Anxiety 27: $507-527$
69. Ferrão YA, Diniz JB, Lopes AC, Shavitt RG, Greenberg B, et al. (2007) Resistance and refractoriness in obsessive-compulsive disorder. Rev Bras Psiquiatr 29 Suppl 2: S66-S76.

70. Insel TR, Mueller EA, Alterman I, Linnoila M, Murphy DL (1985) Obsessivecompulsive disorder and serotonin: is there a connection? Biol Psychiatry 20: 1174-1188.

71. Sichel DA, Cohen LS, Dimmock JA, Rosenbaum JF (1993) Postpartum obsessive compulsive disorder: a case series. J Clin Psychiatry 54: 156-159.

72. Rauch SL, Jenike MA (1993) Neurobiological models of obsessivecompulsive disorder. Psychosomatics 34: 20-32.

73. Zambaldi CF, Cantilino A, Montenegro AC, Paes JA, de Albuquerque TL, et al. (2009) Postpartum obsessive-compulsive disorder: prevalence and clinical characteristics. Compr Psychiatry 50: 503-509.

74. Lensi P, Cassano GB, Correddu G, Ravagli S, Kunovac JL, et al. (1996) Obsessive-compulsive disorder. Familial-developmental history, symptomatology, comorbidity and course with special reference to genderrelated differences. Br J Psychiatry 169: 101-107. 